\title{
1 Novel bacterial lineages associated with boreal moss species
}

2 Hannah Holland-Moritz ${ }^{1,2^{*}}$, Julia Stuart ${ }^{3}$, Lily R. Lewis ${ }^{4}$, Samantha Miller ${ }^{3}$, Michelle C. Mack ${ }^{3}$, Stuart

3 F. McDaniel ${ }^{4}$, Noah Fierer ${ }^{1,2 *}$

4 Affiliations:

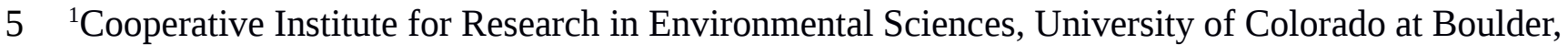

6 Boulder, CO, USA

7 2Department of Ecology and Evolutionary Biology, University of Colorado at Boulder, Boulder, CO,

8 USA

$9{ }^{3}$ Center for Ecosystem Science and Society, Northern Arizona University, Flagstaff, AZ USA

$10{ }^{4}$ Department of Biology, University of Florida, Gainesville, FL 32611-8525, USA

$11{ }^{*}$ Corresponding Author

\section{Abstract}

Mosses are critical components of boreal ecosystems where they typically account for a large proportion of net primary productivity and harbor diverse bacterial communities that can be the major source of biologically-fixed nitrogen in these ecosystems. Despite their ecological importance, we have limited understanding of how microbial communities vary across boreal moss species and the extent to which local environmental conditions may influence the composition of these bacterial communities. We used marker gene sequencing to analyze bacterial communities associated with eight boreal moss species collected near Fairbanks, AK USA. We found that host identity was more important than site in determining bacterial community composition and that mosses harbor diverse lineages of potential $\mathrm{N}_{2^{-}}$ fixers as well as an abundance of novel taxa assigned to understudied bacterial phyla (including candidate phylum WPS-2). We performed shotgun metagenomic sequencing to assemble genomes from the WPS-2 candidate phylum and found that these moss-associated bacteria are likely anoxygenic phototrophs capable of carbon fixation via RuBisCo with an ability to utilize byproducts of photorespiration from hosts via a glyoxylate shunt. These results give new insights into the metabolic capabilities of understudied bacterial lineages that associate with mosses and the importance of plant hosts in shaping their microbiomes. 


\section{Introduction}

Mosses, like their cousins, the vascular plants, associate with a broad diversity of microbes,

31 including, bacteria, fungi, and other microbial eukaryotes (Lindo and Gonzalez, 2010). These moss-

32 microbe associations are particularly relevant to terrestrial nitrogen $(\mathrm{N})$ and carbon $(\mathrm{C})$ cycling in

33 northern ecosystems, where mosses are ubiquitously distributed and can be responsible for as much as

$3450 \%$ of ecosystem net primary productivity (Turetsky et al., 2012). Moss-associated $\mathrm{N}_{2}$-fixing bacteria

35 are often the primary source of ecosystem $\mathrm{N}$ inputs in boreal forests (DeLuca et al., 2002) and moss-

36 associated microbes can have important influences on ecosystem $\mathrm{C}$ dynamics via methane oxidation,

37 especially in peatlands, one of the largest natural sources of atmospheric methane (Kip et al., 2010).

38 Together, the importance of moss-microbial communities to terrestrial biogeochemistry and unique

39 features of bryophyte biology make boreal moss communities a useful system for investigating the

40 interactions between host species identity, microbial community structure, and ecosystem function.

41 Mosses are ubiquitous across boreal forests, with distributions spanning ecologically important

42 environmental gradients (Lindo and Gonzalez, 2010; Turetsky et al., 2012). Moss diversity in these

43 forests can be quite high (Geffert et al., 2013), and different moss species often grow interspersed at a

44 given location, creating many abundant and naturally occurring 'common-garden' experiments for

45 testing how host identity and local environmental conditions influence the assembly of moss-associated

46 microbial communities. Furthermore, mosses, unlike more commonly-studied vascular plants, have

47 simpler leaf-tissue structures and do not possess roots thus representing a comparatively homogeneous

48 host environment, reducing the need to control for inter-tissue spatial variation (a problem when

49 studying vascular plants, e.g. Leff et al., 2015). Mosses are also small enough that an entire plant can

50 be sampled for microbial analyses, an impossible task for most larger plants. Together these traits make

51 mosses a useful study system for investigating the impacts of environmental and host factors on 
52 microbial community structure and the contributions of these moss-associated microbial communities

53 to ecosystem function.

Despite their potential biogeochemical importance and their utility as a model system, we still

55

56

57

58

59

60

61

62

63

64

65

66

67

68

69

70

71

72

73

74

75

know surprisingly little about the structure and function of those microbial communities associated with boreal mosses. Much of the previous research has focused on a handful of abundant boreal moss species, in particular, members of the peat-moss genus Sphagnum, and the dominant feather mosses, Pleurozium schreberi and Hylocomium splendens (e.g. DeLuca et al., 2002; Opelt et al., 2007; Zackrisson et al., 2009; Ininbergs et al., 2011; Bragina, Berg, et al., 2012; Bragina et al., 2015). While these species are among the most abundant mosses in many boreal forests, the microbes living in association with less abundant hosts may be equally as important contributors to key ecosystem processes (Rousk et al., 2015). Previous work on the abundant species makes it clear that boreal mosses possess surprisingly diverse microbial communities, containing not only well-studied Cyanobacteria but also novel and undescribed lineages within the Alphaproteobacteria sub-phylum and the Verrucomicrobia phylum among others (Bragina et al., 2015). However, it is unclear whether local environmental factors govern the microbial community assembly, or whether a moss species hosts a characteristic microbial community, regardless of its abiotic environment.

It is well-known that mosses can harbor $\mathrm{N}_{2}$-fixing bacteria and $\mathrm{N}_{2}$-fixation rates of Sphagnum and the feather mosses, Pleurozium schreberi and Hylocomium splendens, can be quite variable across season (DeLuca et al., 2002), forest type (Zackrisson et al., 2004), and moss species (Leppänen et al., 2015) with reported rates ranging from $0.3-4 \mathrm{~kg} \mathrm{~N} \mathrm{ha}^{-1} \mathrm{yr}^{-1}$ (DeLuca et al., 2007). A number of previous studies have focused on selected moss-associated $\mathrm{N}_{2}$-fixing bacterial taxa, particularly taxa within the Cyanobacteria phylum (reviewed in Rousk, Jones, et al., 2013), and how the composition or diversity of these cyanobacteria relate to $\mathrm{N}_{2}$-fixation rates (Ininbergs et al., 2011). However, Cyanobacteria are unlikely to be the only moss-associated bacteria capable of $\mathrm{N}_{2}$-fixation. For 
76 example, Bragina et al. (2012) found that nitrogenase sequence libraries from bacteria on two

77 Sphagnum species were dominated by alphaproteobacterial sequences, indicating that non-

78 cyanobacterial $\mathrm{N}_{2}$-fixers may play a more important role than previously recognized. In short, it

79 remains unclear which bacteria are responsible for $\mathrm{N}_{2}$-fixation in boreal mosses and how the

80 abundances of these $\mathrm{N}_{2}$-fixing taxa may vary across different moss species.

81 Here we characterized the bacterial communities associated with eight common boreal moss

82 species at three different sites using marker gene (16S rRNA gene) amplicon sequencing. We used this

83 dataset to address the following questions: 1) How do bacterial communities vary across different moss

84 species and different sites? and 2) Which microbial taxa from these communities are potential $\mathrm{N}_{2^{-}}$

85 fixers? Based on our observation that many of the taxa found in association with the moss species were

86 representatives of bacterial lineages for which little is known (including members of the candidate

87 phylum WPS-2), we then assembled genomes from shotgun metagenomic data to determine the

88 functional attributes of these abundant, ubiquitous, and previously undescribed members of moss-

89 associated microbial communities.

\section{Experimental Procedures}

\section{Sample Collection}

To characterize and compare the bacterial communities associated with different moss host species

93 and their potential contributions to $\mathrm{N}_{2}$-fixation, we collected samples of eight boreal moss species (the

94 closely related pleurocarpous mosses Pleurozium schreberi (Brid.) Mitt., Hylocomnium splendens

95 (Hedw.) Shimp, Sanionia uncinata (Hedw.) Loeske, and Tomenthypnum nitens (Hedw.) Loeske, and the

96 successively more distantly related species, Aulacomnium palustre (Hedw.) Schwägr. and A. turgidum

97 (Wahlenb.) Schwägr., Dicranum elongatum Schleich. ex Schwägr., and Sphagnum capillifolium (Ehrh.)

98 Hedw.). The samples were collected from three black spruce (Picea mariana)-dominated sites that were 
99 each at least a kilometer apart within the arboretum of the University of Alaska Fairbanks during late

100 July 2014. The selected moss species are both common in boreal forests and represent lineages

101 spanning the phylogenetic diversity of mosses. At each of the three sites we collected one sample for

102 each of the eight species (except Aulacomnium turgidum and Dicranum elongatum, which could only

103 be found at two sites), resulting in a total of 22 samples for analysis. Each sample was divided into four

104 subsamples of ten stems each for microbial community analysis, isotopic enrichment, natural

105 abundance voucher, and taxonomic voucher specimens (See Table S1). For each species within each

106 site, a clump of ramets were removed from a monospecific patch and carefully sorted and cleaned with

107 a gloved hand. Brown or decaying material was removed from the bottom so that each ramet was

108 approximately $5 \mathrm{~cm}$ in length and included the apical meristem. Each sample was then divided into

109 four subsamples of ten stems each for microbial community analysis, isotopic enrichment, natural

110 abundance voucher, and taxonomic voucher specimens (See Table S1). Samples collected for microbial

111 analyses were placed in a cooler on blue ice in the field and returned to the lab where they were frozen

$112\left(-20^{\circ} \mathrm{C}\right)$ within $2 \mathrm{~h}$ of collection. Samples for the $\mathrm{N}_{2}$-fixation rate assays were also placed in the coolers

113 until the measurements were started, usually within $2 \mathrm{~h}$ of sample collection. Natural abundance

114 voucher specimens were returned to the lab and dried at $60^{\circ} \mathrm{C}$ for $48 \mathrm{~h}$ within $2 \mathrm{~h}$ of collection.

\section{$115 \quad \mathbf{N}_{2}$-Fixation Measurements}

116 To quantify field rates of $\mathrm{N}_{2}$-fixation for each sample, we used an isotopic enrichment approach

117 modified from Ruess et al. (2009, 2013). Each sample was placed in a $60 \mathrm{~mL}$ translucent polycarbonate

118 syringe that was depressed to contain $10 \mathrm{ml}$ of air. We then added $10 \mathrm{~mL}$ of ${ }^{15} \mathrm{~N}_{2}$ (98\% enriched,

119 Cambridge Isotope Laboratories, Inc., U.S.A.) and sealed the syringe with a stopcock. Sealed syringes

120 from all sites were placed in site A, where they were incubated for $24 \mathrm{~h}$ under similar light and

121 temperature conditions. After $24 \mathrm{~h}$, mosses were removed from the syringes and placed in a $60{ }^{\circ} \mathrm{C}$ oven

122 for 48 h. Both enriched incubation samples and natural abundance samples were ground to a 
123 homogenous powder. Nitrogen and carbon concentrations and atom $\%{ }^{15} \mathrm{~N}$ and ${ }^{13} \mathrm{C}$ values for both

124 enriched incubation samples and natural abundance voucher specimens were measured on a Costech

125 ECS4010 coupled to a Thermo Scientific Delta V Advantage Isotope ratio mass spectrometer in Mack’s

$126 \mathrm{lab}$ at Northern Arizona University. Nitrogen fixation was calculated by comparing the ${ }^{15} \mathrm{~N}$ values from

127 enriched and control samples (see Jean et al. (2017) for details).

\section{Amplicon-Based Bacterial Community Analysis}

129 To analyze the bacterial communities associated with each of the collected moss specimens, we

130 PCR amplified and sequenced a portion of the bacterial and archaeal 16S rRNA marker gene. First, to

131 minimize any bias from micro-spatial differences along the moss tissue, we homogenized each sample

132 (ten moss stems per sample, approximately $0.25 \mathrm{~g}$ of tissue) with liquid $\mathrm{N}_{2}$ under sterile conditions. We

133 then extracted DNA from each homogenized moss sample using the MoBio Power Soil DNA extraction

134 kit (MoBio Laboratories, Carlsbad, CA). After extracting DNA, we used the 515f / 806r primers to

135 PCR amplify the V4-V5 region of the 16S rRNA gene (Caporaso et al., 2012). For each sample, we

136 used a unique primer pair that included a 12-bp barcode and Illumina sequencing adapters to allow for

137 multiplexed sequencing. To minimize amplification of mitochondrial and chloroplast DNA, we used

138 PNA (peptide nucleic acid) clamps during PCR amplification (Lundberg et al., 2013). During both

139 DNA extraction and PCR amplification, we included negative controls to check for potential

140 contaminants introduced during those steps. We prepared the samples for sequencing by normalizing

141 the concentrations of PCR products across all samples using the ThermoFisher Scientific SequalPrep

142 Normalization plate (Thermo Fisher Scientific Inc. USA) and pooled the amplicons together. We

143 sequenced all samples on the Illumina MiSeq platform running the 2 x 150 bp paired-end chemistry at

144 the University of Colorado Next Generation Sequencing Facility. The Hylocomium splendens

145 amplicon sequences were sequenced on a separate run. Because we cannot control for run-to-run

146 variation we have excluded these samples from downstream statistical analyses. A cursory analysis 
147 indicates that the Hylocomnium microbiome is statistically distinguishable from the other mosses in our

148 sample, however the community was not marked by novel species relative to the other samples we 149 analyzed.

150 We used the approach described by Leff et al. (2015) to analyze the 16S rRNA sequence data.

151 Briefly, we removed adapters from the raw reads using cutadapt (paired, -O 1) (Martin, 2011) and

152 demultiplexed the reads using a custom in-house python script (“prep_fastq_for_uparse_paired.py” at:

153 https://github.com/leffj/helper-code-for-uparse/). Then, using USEARCH v.8 (Edgar, 2010), we

154 merged, quality filtered ("maxee rate” = 0.005) and dereplicated the reads to create a fasta file

155 containing each unique amplicon sequence. We also removed singletons (sequences found only once

156 across the entire sample set) from the dereplicated reads. We created a de novo database from our

157 sequences using the UPARSE pipeline (Edgar, 2013) (implemented with USEARCH v.8) by clustering

158 reads at $\geq 97 \%$ sequence similarity level (which we refer to as 'phylotypes'). As a quality-control

159 measure, we removed representative sequences from phylotypes that were less than $75 \%$ similar to any

160 sequence in the Greengenes database (version August 2013) (McDonald et al., 2012). We assumed

161 these highly divergent sequences to be chimeric, a product of non-specific amplification, or of

162 insufficient quality.

163 To generate phylotype counts, we mapped the merged reads back to the de novo database

164 (command “-usearch_global”, -id 0.97) and used a custom python script

165 (“create_otu_table_from_uc_file.py” at: https://github.com/leffj/helper-code-for-uparse/) to generate a

166 table of phylotype counts per sample from the USEARCH mapping output. We classified the reads

167 with the RDP Naïve Bayesian classifier (Wang et al., 2007) against the Greengenes database

168 (McDonald et al., 2012) and removed any residual chloroplast and mitochondrial sequences. To control

169 for differences in read depth (sequencing “effort”) across samples, we randomly selected 6000 bacterial

170 and archaeal 16S rRNA gene reads per sample prior to downstream analyses. The 6000 read cut-off 
171 was chosen based on the read depth of the sample with fewest reads after mitochondria and chloroplast

172 removal. The filtered reads can be found on FigShare (DOI:

173 https://doi.org/10.6084/m9.figshare.5594527).

174 To measure differences between communities across host species and sites, we calculated pairwise

175 Bray-Curtis dissimilarities on the square-root transformed phylotype table and tested for differences

176 between species and sites using a PERMANOVA test. All statistical analyses were conducted in R (R

177 Core Team, 2017, package “Vegan”, R Core Team, 2017). We used metaMDS (R package “Vegan”) to

178 generate NMDS plots from the dissimilarity matrix.

\section{Identifying Nearest Isolated Representatives and Classifying Putative $\mathbf{N}_{2}$-fixing Bacteria}

180 To identify the nearest isolated representatives of the 30 most abundant bacterial phylotypes, we

181 used the RDP (Ribosomal Database Project) SeqMatch tool which finds the nearest relatives by

182 comparing the percent of shared sub-sequences between a query and the RDP isolate database (Cole et

183 al., 2014). We then downloaded the nearest-neighbor representative sequences from RDP and used

184 MUSCLE (Edgar, 2004) to create an alignment of the isolate sequences and representative sequences

185 from the 30 most abundant bacterial phylotypes, using FastTree (Price et al., 2010) to generate the final

186 tree. We defined "putative $\mathrm{N}_{2}$-fixing phylotypes" as those phylotypes whose nearest relative ( $\geq 97 \%$

187 similarity in the $16 \mathrm{~S}$ rRNA gene region) has been found to be capable of $\mathrm{N}_{2}$-fixation when cultivated in

188 isolation under laboratory conditions (four phylotypes). Alternatively, if the nearest neighbor was <

189 97\% similar but came from a lineage which included only previously-described representative strains

190 known to be capable of $\mathrm{N}_{2}$-fixation, the phylotype was also defined as a "putative $\mathrm{N}_{2}$-fixer" (two

191 phylotypes). 


\section{Shotgun Metagenomic Sequencing and Analysis}

Based on the preponderance of bacterial lineages identified from the 16S rRNA amplicon analyses

194

195

196

197

198

199

200

201

202

203

204

205

206

207

208

209

210

211

212

213 Salzberg, 2012) to map the filtered reads back to each bin. We used CheckM (Parks et al., 2015) to

214 verify the completeness and contamination of each bin (Table S2). Briefly, CheckM measures

215 metagenomic completeness and contamination on the basis of presence and number of conserved

216 single-copy marker genes (genes which typically are present in bacterial genomes only once). The 
217 number of marker genes present compared to the number of expected marker genes for a particular

218 bacterial lineage is used as a measure of completeness while the number of copies of a marker gene

219 indicates contamination. We considered bins that were greater than $70 \%$ complete and less than $10 \%$

220 contaminated to be "high-quality” bins (as per the top two levels of quality identified in Parks et al.

221 (2015).

222 Because few of our high-quality bins contained assembled 16S rRNA sequences (a common 223 problem, see Miller et al., 2011), we used three separate methods to confirm the taxonomic identity of 224 bins. First, we used the program EMIRGE (Miller et al., 2011) to assemble full-length 16S rRNA 225 sequences from the raw metagenomic reads and used USEARCH (Edgar, 2010) to match contigs from 226 the bins to the EMIRGE-assembled full-length 16S rRNA sequences. Second, to verify this result with 227 a technique that did not rely on genome assembly from metagenomic data, we used Metaxa2

228 (Bengtsson-Palme et al., 2015) to independently identify fragments of the 16S rRNA gene in the 229 binned contigs and classified those fragments against the Greengenes database using USEARCH 230 (Edgar, 2010). Finally, we used the automatically-generated concatenated marker gene phylogeny 231 available from CheckM to confirm that bins were clustered on a tree in the appropriate clade as 232 indicated by analysis of their 16S rRNA gene sequences. After identifying the organisms represented 233 by the bins, we used the Joint Genome Institute’s Integrated Microbial Genomes (IMG) analysis 234 pipeline (Markowitz et al., 2014) to identify and assign functions to the genes in the assembled bins. 235 The assembled metagenomic reads are available through the IMG portal (IMG submission ID 115847).

\section{Results and Discussion}

\section{Moss Species Identity Drives Microbiome Composition}

Our analyses of moss-associated bacterial 16S rRNA amplicons show that mosses host diverse and 239 species-specific bacterial communities. After quality filtering, but prior to chloroplast and 
240 mitochondrial filtering, we obtained 7947 to 1659016 S rRNA gene reads per sample with an average

241 sequencing depth of 11430 reads. The chloroplast and mitochondrial sequences that were removed

242 made up only a small percentage of the reads (10\% and 5\%, respectively). Samples had an average

243 richness of 924 phylotypes per sample and were dominated by eight bacterial phyla. Archaeal

244 sequences were extremely rare and made up less than $0.001 \%$ of all reads. We found similar archaeal

245 abundances in the primer-independent metagenomic data suggesting that the low archaeal abundance in

246 the amplicon data was not a product of primer biases. The dominant bacterial phyla, as measured by

247 average read abundance across all samples, were Proteobacteria (44.8\% of reads across all samples),

248 Acidobacteria (10.8\%), Verrucomicrobia (9.8\%), Bacteroidetes (9.3\%), Cyanobacteria (6.5\%),

249 Candidate phyla WPS-2 (5.7\%), Planctomycetes (5.2\%), and Actinobacteria (4.2\%) (Figure 1). The

250 most abundant phylotypes identified across the entire sample set included those assigned to the

251 Acetobacteraceae (9.5\%), Acidobacteriaceae (8.2\%), Sinobacteraceae (8.2\%) and Nostocaceae (5.2\%)

252 families as well as many phylotypes that could only be classified to the phylum or class level of

253 resolution (including those in the WPS-2, and Verrucomicrobia phyla, Figure 1). These phyla and

254 families have been found in other microbial studies of mosses; notably Acetobacteraceae and

255 Acidobacteriaceae were found to be abundant in sphagnum mosses (Bragina, Berg, et al., 2012) and

256 Nostocaceae have long been studied in association with feather mosses(DeLuca et al., 2002) and

257 Sphagnum (Bay et al., 2013; Kostka et al., 2016). Members of the WPS-2 and Verrucomicrobia phyla

258 have previously been found in association with sphagnum mosses in bogs (Bragina et al., 2015).

259 Moss species identity was more important than site in determining the composition of the moss-

260 associated bacterial communities. Across all samples, moss species contributed to 63\% of the variation

261 in community composition (Permanova $\mathrm{R}^{2}=0.63, \mathrm{p}<0.001$ ), while site was not a significant source of

262 variation (Permanova $\mathrm{R}^{2}=0.076, \mathrm{p}=0.21$ ) (Figure 2). This finding is consistent with studies in

263 vascular plants which find that species identity is generally more important than site in shaping

264 phyllosphere community composition (Redford et al., 2010; Laforest-Lapointe et al., 2016). However, 
265

266

267

268

269

270

271

272

273

274

275

276

277

278

279

280

281

282

283

284

285

286

287

288

sites in this study were situated within a relatively small area. It is possible that across larger scales, differences in local environmental conditions may have a stronger influence on the observed bacterial communities. Across all moss species studied here, Sanionia uncinata harbored particularly distinct bacterial communities. More than half (59\%) of phylotypes found on Sanionia uncinata were not found in any of the other moss species and, of the 30 most abundant phylotypes highlighted in Table S3, only $77 \%$ were present on S. uncinata compared to more than $90 \%$ found across every other species (Figure 3). While most of the mosses were dominated by Acetobacteraceae (Alphaproteobacteria),

Acidobacteriaceae (Acidobacteria), and Methylacidiphilales (Verrucomicrobia), the moss species

Sanionia uncinata had a low abundance or complete absence of these taxa. Instead, Sanionia uncinata was dominated by Comamonadaceae (Betaproteobacteria), Nostocacaceae (Cyanobacteria) and Chitinophagageae (Bacteroidetes). The distinctiveness of the S. uncinata microbiome does not appear to reflect any aspect of phylogenetic relatedness among the sampled moss species. For instance, the pleurocarpous $S$. uncinata has much closer phylogenetic affinities with $P$. schreberi and T. nitens, yet the phylogenetically distant Sphangnum capillifolium hosts a microbiome much more similar to $P$. schreberi and T. nitens than does S. uncinata. The factors driving these differences between S. uncinata and the other moss species remain uncertain.

\section{Evidence for multiple groups of $\mathbf{N}_{2}$-fixing bacteria in boreal mosses}

Using stable-isotope enrichment, we measured $\mathrm{N}_{2}$-fixation rates and found that all of the moss species were actively fixing $\mathrm{N}_{2}$ (Figure S1), though rates varied by a factor of 100 across samples (from 0.98 to $100 \mu \mathrm{g} \mathrm{N} \cdot \mathrm{g}$ dry weight moss $^{-1}$ day $^{-1}$ ). Importantly, we find that many moss microbiomes which were assumed to be non- $\mathrm{N}_{2}$-fixing (the genus Dicranum, for example, Gundale et al. (2011) do, in fact, appear to associate with bacteria capable of fixing $\mathrm{N}_{2}$ at rates comparable to those of feather mosses, which are well-known to host bacteria capable of sustaining relatively high rates of $\mathrm{N}_{2}$-fixation (DeLuca et al., 2002; Turetsky et al., 2012). Consistent with other studies, Pleurozium schreberi and 
289 Sanionia uncinata had the highest average rates of $\mathrm{N}_{2}$-fixation at 46.1 and $52.4 \mu \mathrm{g} \mathrm{N} \cdot \mathrm{g}$ dry weight

290 moss $^{-1}$ day $^{-1}$, respectively. Our measured rates of fixation are within the expected range previously

291 reported for Sphagnum mosses and Pleurozium schreberi (Vile et al., 2014). However, because we used

292 a $\partial^{15} \mathrm{~N}$ method as opposed to an acetylene reductase assay, and because of the lack of reported $\mathrm{N}_{2}$

293 fixation studies for some of the moss species in our study, it is difficult to directly compare all of our

294 measurements of $\mathrm{N}_{2}$-fixation rates to those reported previously (e.g. DeLuca et al., 2002; Zackrisson et

295 al., 2004; Gundale et al., 2012).

296 Given that all of the sampled moss species had microbial communities with measurable $\mathrm{N}_{2}$-fixation 297 activities, we then used the 16S rRNA gene survey data to determine which taxa might be contributing 298 to the $\mathrm{N}_{2}$-fixation. We found three cyanobacterial and three alphaproteobacterial lineages that were

299 reasonably abundant across nearly all moss species, with all of these lineages having previously been 300 identified as capable of $\mathrm{N}_{2}$-fixation. Specifically, the putative $\mathrm{N}_{2}$-fixers in our samples were from the

301 Nostocaeae, Acetobacteraceae, Bradyrhizobiaceae, and Methylocystaceae families (Figure 3). Previous

302 studies have focused on the role of Cyanobacteria in bryophyte-associated $\mathrm{N}_{2}$ - fixation, but one study

303 in Sphagnum, found that 50\% of bacterial cells colonizing Sphagnum were from Alphaproteobacteria

304 and that the nifH gene libraries for these species were dominated by alphaproteobacterial, rather than

305 cyanobacterial sequences (Bragina, Maier, et al., 2012). Interestingly, these two groups of putative $\mathrm{N}_{2-}$

306 fixers showed contrasting patterns of abundance between Sanionia uncinata and Pluerozium schreberi

307 (the two moss species with the highest rates of $\mathrm{N}_{2}$-fixation). These two hosts showed relatively similar

308 average rates of $\mathrm{N}_{2}$-fixation (52.4 $\mu \mathrm{g} \mathrm{N} \mathrm{g}^{-1} \mathrm{day}^{-1}$ and $46.1 \mu \mathrm{gN} \mathrm{g}^{-1} \mathrm{day}^{-1}$ ), yet Sanionia uncinata was

309 dominated by Cyanobacteria (79\%) and showed low relative abundances of the alphaproteobacterial

$310 \quad \mathrm{~N}_{2}$-fixers (7.2\%), while Pleurozium schreberi showed the opposite pattern (6.8\% cyanobacterial, $21.4 \%$

311 alphaproteobacterial). Together, these results suggest that multiple bacterial taxa may contribute to the

312 measured $\mathrm{N}_{2}$-fixation activity in these boreal moss species and, even though cyanobacterial $\mathrm{N}_{2}$-fixers

313 have received the bulk of the attention in previous studies (e.g. Ininbergs et al., 2011; Rousk, Rousk, et 
314 al., 2013), they are not the only $\mathrm{N}_{2}$-fixing bacteria that associate with boreal mosses. To identify which

315 of these putative $\mathrm{N}_{2}$-fixing lineages are responsible for the measured $\mathrm{N}_{2}$-fixation activities in these

316 moss species, future work using stable isotope probing-based approaches would be necessary (e.g.

317 Buckley et al., 2007; Jehmlich et al., 2010).

\section{Boreal mosses harbor abundant, undescribed bacterial lineages}

The mosses we studied hosted an unexpected abundance of understudied bacterial lineages.

320 Abundant phylotypes for which there were no close matches (<97\% 16S rRNA sequence similarity) to

321 previously cultivated and described bacteria are summarized in Table S1. Of these phylotypes, several

322 of the most abundant phylotypes were from the phylum Verrucomicrobia and candidate phylum WPS-

323 2. These phylotypes had no closely related cultivated representatives beyond the phylum-level of

324 resolution, yet they were consistently among the most abundant phylotypes in many of the samples

325 (Table S1, Figure S2). Further analysis of the metagenomic data confirmed the observed high relative

326 abundances of WPS-2 and Verrucomicrobia and highlight that the abundances observed with the

327 amplicon-based analyses were not a product of primer biases (Figure S3).

328 Verrucomicrobia are widely distributed in soils (Brewer et al., 2016), acidic geothermal

329 environments (Op den Camp et al., 2009), and have also been found in boreal mosses, particularly in

330 those mosses growing in moist, acidic environments such as bogs and peatlands (Dedysh, 2011; Sharp

331 et al., 2014; Bragina et al., 2015). However, the ecologies of these moss-associated taxa remain poorly

332 known. Among our samples, the most common verrucomicrobial divisions were the orders

333 Methylacidiphilales (3.24\%) and Spartobacteria (3.79\%). One phylotype from Methylacidiphilales,

334 was the third most abundant phylotype overall. Several earlier studies hypothesized that bacteria from

335 Methylacidiphilales contribute to both $\mathrm{N}_{2}$-fixation and methanotrophy in peatland environments

336 (Bragina et al., 2015; Ho and Bodelier, 2015). We searched the assembled metagenomic contigs for the

337 methanotrophic marker gene ( $p m o A)$, and we were unable to find any evidence of the methanotrophic 
338

339

340

marker gene $(p m o A)$ associated with Methylacidiphilales in our metagenomic sequence data. It is therefore unlikely that Methylacidiphilales in these samples are oxidizing methane unless they are using genes that are not currently recognized in databases as being linked to methane oxidation.

In addition to relatively high abundances of poorly-described verrucomicrobial phylotypes, the mosses were also dominated by a single phylotype from the candidate phylum WPS-2 which represented 3.5\% of the 16S rRNA sequences analyzed (Figure 3), making it one of the most abundant phylotypes in six of the seven moss species (with the exception of Sanionia uncinata). No members of WPS-2 have ever been cultured, and no published genome exists for any member of this candidate phylum. Given how little we know about this phylum, we can only speculate about the ecology of this group from a few previous studies in which members of this phylum have been detected.

Representatives from WPS-2 have previously been found in acidic and cold environments, including alpine bog vegetation (Bragina et al., 2015), mineral deposits from a low-temperature acidic spring (Grasby et al., 2013), and acidic natural gas extraction shale (Trexler et al., 2014). Interestingly, Trexler et al. (2014) also observed that WPS-2 seems to co-occur with methanotrophs in aquatic mosses and speculated that WPS-2 may be using derivatives of methanotrophy such as carbon dioxide, formaldehyde, or formate. However, we found only two instances of the methane oxidation marker gene $(p m o A)$ in our shotgun metagenomes (out of more than 926 million reads), therefore it seems unlikely that the highly abundant members of the WPS-2 phylum found in our samples associate with methanotrophs.

\section{Draft genome of WPS-2 recovered from metagenomic data}

To learn more about the abundant and poorly studied taxa found in these mosses and to provide further insight into the functional attributes of these bacteria, we chose moss samples with particularly high abundances of diverse taxa of interest (Methylacidiphilae and WPS-2) for metagenomic analysis. We assembled 61 genome bins from these moss-associated bacterial communities, seven of which 
362 passed contamination and completeness standards (Table S2). Despite the apparent abundance of

363 Methylacidiphilae, none of the recovered bins were from this phylum. The inability to recover highly

364 abundant strains from metagenomes is fairly common and can be a product of high strain variation

365 (intraspecific variation) (Miller et al., 2011).

366

Four phyla were represented in the recovered genome bins: Acidobacteria (1 bin), Proteobacteria

367 (1 bin, Alphaproteobacteria), Cyanobacteria (2 bins), and WPS-2 (3 bins). The three bins from WPS-2

368 ranged from 84 - 89\% complete and represent the first genomic data available for members of the

369 candidate phylum WPS-2. We estimate that the full genome sizes for these WPS-2 representatives are

370 3.88, 3.41, and 4.34 Mbp based on the presence of lineage-specific single-copy marker genes.

371 Because WPS-2 is one of the most abundant groups across our samples and we know so little about

372 the functional attributes of this group, we used gene annotation to try to reconstruct the potential

373 metabolic attributes of the most complete genome bin obtained for WPS-2. The gene annotations

374 revealed that WPS-2 is likely an anoxygenic phototroph, capable of carbon fixation, and able to

375 metabolize the bi-products of photorespiration making it well-suited to life on the surface of a plant

376 (Figure 4). We identified key genes involved in anoxygenic photosynthesis including those encoding

377 the $\mathrm{M}$ and $\mathrm{L}$ subunits of anoxygenic photosynthetic reaction centers (pufM and pufL) as well as the

378 gene for the Y subunit of chlorophyllide a reductase (bchY), a universal marker gene for BChl-

379 containing anoxygenic phototrophs (Yutin et al., 2005, 2009). Anoxygenic photosynthesis was first

380 recognized for its importance in marine environments, but recently it has also been recognized as a

381 common trait of phyllosphere bacteria found on the surface of vascular plants (Atamna-Ismaeel et al.,

382 2013). There are three types of anoxygenic phototrophs that contain pufM-type reaction centers: purple

383 sulfur bacteria, purple non-sulfur bacteria, and aerobic anoxygenic phototrophs (AAP) (Yurkov and

384 Hughes, 2017). Of these three groups, we hypothesize that WPS-2 is an aerobic anoxygenic phototroph

385 since abundant sulfur sources are expected to be extremely limited in boreal forests and the exposed 
environment of the moss phyllosphere is unlikely to provide the anaerobic environment necessary to support purple non-sulfur bacteria. If WPS-2 is truly an AAP, it would be one of the few observed lineages to possess RuBisCo. Until recently, all other known AAP were thought incapable of RuBisCofacilitated carbon fixation (Hughes et al., 2017) however, a recent study (Graham et al., 2017) has found four Alphaproteobacteria in a marine metagenomic dataset that appear to possess the ability to fix carbon via the Calvin-Benson-Bassham cycle.

If WPS-2 is an AAP possessing RuBisCo, how might it live in close association to moss and their cyanobacteria without being out-competed for light and carbon resources? WPS-2 appears to possess several traits which may allow it to effectively associate with mosses. As an anoxygenic phototroph, WPS-2 would not compete with its host for light resources since anoxygenic phototrophs absorb light in a complementary spectrum to that of plants and cyanobacteria, with a maximal absorption peak between 500 and 550nm (the region where chlorophyll has its minimum absorption) (Atamna-Ismaeel et al., 2012). Interestingly, an absorption peak in this area of the spectrum may also give WPS-2 a photosynthetic advantage under light snow cover since the wavelengths with the greatest transmittance through snow are also in that region of the light spectrum (Perovich, 2007).

In addition to its complementary absorption spectrum, WPS-2 may be able to take advantage of the 402 byproducts of its host's photorespiration to supplement its C requirements. Photorespiration takes place 403 when RuBisCo acts on $\mathrm{O}_{2}$ rather than $\mathrm{CO}_{2}$ producing phosphoglycolate, a potent inhibitor of the Calvin 404 cycle. Thus, in plants and other carbon fixers who use the Calvin cycle, metabolizing phosphoglycolate 405 is important. The moss-associated WPS-2 phylotypes appear to possess the enzymes necessary for converting phosphoglycolate into glyoxylate and passing it into the TCA cycle. This process, known as 407 the "glyoxylate bypass", is a modified TCA cycle that allows organisms to bypass the normal electron408 generating steps of the cycle and devote it entirely to biomass production (Kornberg, 1966). Since 409 WPS-2 is likely phototrophic, during times of high light it is unlikely to need its TCA cycle for energy 
410 generation and could instead take advantage of its host's sugars for biomass production. In such a

411 situation, WPS-2 would only need to use RuBisCo under conditions when sugars from the plants were

412 scarce, such as early in the growing season or under snow pack. The complementary absorption

413 spectrum of the moss-associated members of the WPS-2 phylum and their ability to metabolize

414 glyoxylate appear to make this group uniquely equipped for living commensally on their moss hosts.

\section{Conclusions}

We found that moss-associated bacterial communities are strongly structured by moss species

417 identity with different moss species harboring distinct bacterial communities regardless of collection

418 site. Surprisingly, all of the moss species studied possessed microbial communities that were capable of

$419 \mathrm{~N}_{2}$-fixation and hosted a broad diversity of putative $\mathrm{N}_{2}$-fixing bacterial lineages. However, it remains

420 unclear which lineages are responsible for the measured $\mathrm{N}_{2}$-fixation rates and whether distinct bacterial

421 lineages are driving $\mathrm{N}_{2}$-fixation in different moss species. Future work should focus on how microbial

422 community variation between host species may influence $\mathrm{N}_{2}$-fixation rates and how interactions both

423 between bacteria and between bacteria and the moss host might contribute to these rates. We also found

424 that mosses can harbor a number of poorly-described bacterial lineages, including a high relative

425 abundance of bacteria assigned to the candidate phylum WPS-2 with previously unknown ecological

426 and metabolic characteristics. Using shotgun metagenomic analyses, we were able to assemble a nearly

427 complete genome representative of this WPS-2 lineage and the genomic analyses suggest that WPS-2 is

428 an anoxygenic phototroph that is uniquely adapted to living in close-association with mosses in this

429 ecosystem.

\section{Acknowledgements}

431 Financial support for this work was provided by the U.S. National Science Foundation Dimensions of

432 Biodiversity program (DEB 1542609). The authors acknowledge infrastructural support provided by

433 the University of Colorado Next Generation Sequencing Facility and the University of Alaska,

434 Fairbanks. Helpful feedback on the manuscript was provided by the University of Colorado, EBIO

435 writing co-op. 


\section{References}

Atamna-Ismaeel, N., Finkel, O., Glaser, F., von Mering, C., Vorholt, J.A., Koblížek, M., et al. (2012) Bacterial anoxygenic photosynthesis on plant leaf surfaces. Environ. Microbiol. Rep. 4: 209-216.

Atamna-Ismaeel, N., Finkel, O.M., Glaser, F., Sharon, I., Schneider, R., Post, A.F., et al. (2013) Microbial rhodopsins on leaf surfaces of terrestrial plants. Environ. Microbiol. 14: 140-146.

Bay, G., Nahar, N., Oubre, M., Whitehouse, M.J., Wardle, D.A., Zackrisson, O., et al. (2013) Boreal feather mosses secrete chemical signals to gain nitrogen. New Phytol. 200: 54-60.

Baym, M., Kryazhimskiy, S., Lieberman, T.D., Chung, H., Desai, M.M., and Kishony, R.K. (2015) Inexpensive multiplexed library preparation for megabase-sized genomes. PLoS One 10: 1-15.

Bengtsson-Palme, J., Hartmann, M., Eriksson, K.M., Pal, C., Thorell, K., Larsson, D.G.J., and Nilsson, R.H. (2015) metaxa2: Improved identification and taxonomic classification of small and large subunit rRNA in metagenomic data. Mol. Ecol. Resour. 15: 1403-1414.

Bragina, A., Berg, C., and Berg, G. (2015) The core microbiome bonds the Alpine bog vegetation to a transkingdom metacommunity. Mol. Ecol. 24: 4795-4807.

Bragina, A., Berg, C., Cardinale, M., Shcherbakov, A., Chebotar, V., and Berg, G. (2012) Sphagnum mosses harbour highly specific bacterial diversity during their whole lifecycle. ISME J. 6: 802-13.

Bragina, A., Maier, S., Berg, C., Müller, H., Chobot, V., Hadacek, F., and Berg, G. (2012) Similar diversity of Alphaproteobacteria and nitrogenase gene amplicons on two related Sphagnum mosses. Front. Microbiol. 2: 1-10.

Brewer, T., Handley, K., Carini, P., Gibert, J., and Fierer, N. (2016) Genome reduction in an abundant and ubiquitous soil bacterium “Candidatus Udaeobacter copiosus.” Nat. Microbiol. 2: 16198.

Buckley, D.H., Huangyutitham, V., Hsu, S.F., and Nelson, T.A. (2007) Stable isotope probing with ${ }^{15}$ N2 reveals novel noncultivated diazotrophs in soil. Appl. Environ. Microbiol. 73: 3196-3204.

Caporaso, J.G., Lauber, C.L., Walters, W.A., Berg-Lyons, D., Huntley, J., Fierer, N., et al. (2012) Ultrahigh-throughput microbial community analysis on the Illumina HiSeq and MiSeq platforms. ISME J. 6: 1621-1624.

Cole, J.R., Wang, Q., Fish, J.A., Chai, B., McGarrell, D.M., Sun, Y., et al. (2014) Ribosomal Database Project: data and tools for high throughput rRNA analysis. Nucleic Acids Res. 42: 633-642.

Dedysh, S.N. (2011) Cultivating uncultured bacteria from northern wetlands: Knowledge gained and remaining gaps. Front. Microbiol. 2: 1-15.

DeLuca, T.H., Zackrisson, O., Gentili, F., Sellstedt, A., and Nilsson, M.C. (2007) Ecosystem controls on nitrogen fixation in boreal feather moss communities. Oecologia 152: 121-130.

DeLuca, T.H., Zackrisson, O., Nilsson, M.-C., and Sellstedt, A. (2002) Quantifying nitrogen-fixation in feather moss carpets of boreal forests. Nature 419: 917-920.

Edgar, R.C. (2004) MUSCLE: Multiple sequence alignment with high accuracy and high throughput. Nucleic Acids Res. 32: 1792-1797. 
Edgar, R.C. (2010) Search and clustering orders of magnitude faster than BLAST. Bioinformatics 26: 2460-2461.

Edgar, R.C. (2013) UPARSE: highly accurate OTU sequences from microbial amplicon reads. Nat. Methods 10: 996-8.

Geffert, J.L., Frahm, J.P., Barthlott, W., and Mutke, J. (2013) Global moss diversity: spatial and taxonomic patterns of species richness. J. Bryol. 35: 1-11.

Graham, E., Heidelberg, J.F., and Tully, B. (2017) Undocumented Potential For Primary Productivity In A Globally-Distributed Bacterial Photoautotroph. bioRxiv.

Grasby, S.E., Richards, B.C., Sharp, C.E., Brady, A.L., Jones, G.M., Dunfield, P.F., and Williamson, M.-C. (2013) The Paint Pots, Kootenay National Park, Canada - a natural acid spring analogue for Mars 1, 2. Can. J. Earth Sci. 50: 94-108.

Gundale, M.J., Deluca, T.H., and Nordin, A. (2011) Bryophytes attenuate anthropogenic nitrogen inputs in boreal forests. Glob. Chang. Biol. 17: 2743-2753.

Gundale, M.J., Nilsson, M., Bansal, S., and Jäderlund, A. (2012) The interactive effects of temperature and light on biological nitrogen fixation in boreal forests. New Phytol. 194: 453-463.

Ho, A. and Bodelier, P.L.E. (2015) Diazotrophic methanotrophs in peatlands: the missing link? Plant Soil 419-423.

Hughes, E., Head, B., Maltman, C., Piercey-Normore, M., and Yurkov, V. (2017) Aerobic anoxygenic phototrophs in gold mine tailings in. Can. J. Microbiol. 63: 212-218.

Ininbergs, K., Bay, G., Rasmussen, U., Wardle, D.A., and Nilsson, M.C. (2011) Composition and diversity of nifH genes of nitrogen-fixing cyanobacteria associated with boreal forest feather mosses. New Phytol. 192: 507-517.

Jean, M. (2017) Broadleaf litter and environmental effects on bryophytes in boreal forests of Alaska.

Jehmlich, N., Schmidt, F., Taubert, M., Seifert, J., Bastida, F., von Bergen, M., et al. (2010) Proteinbased stable isotope probing. Nat. Protoc. 5: 1957-1966.

Joshi, N.A. and Fass, J.N. (2011) Sickle: A sliding-window, adaptive, quality-based trimming tool for FastQ files (Version 1.33) [Software].

Kip, N., Winden, J.F. Van, Pan, Y., Bodrossy, L., Reichart, G., Smolders, A.J.P., et al. (2010) Global prevalence of methane oxidation by symbiotic bacteria in peat-moss ecosystems. Nat. Geosci. 3: 617-621.

Kornberg, H.L. (1966) The role and control of the glyoxylate cycle in Escherichia coli. Biochem. J. 99: $1-11$.

Kostka, J.E., Weston, D.J., Glass, J.B., Lilleskov, E.A., Shaw, A.J., and Turetsky, M.R. (2016) Tansley insight The Sphagnum microbiome: new insights from an ancient plant lineage. New Phytol. 
Laforest-Lapointe, I., Messier, C., and Kembel, S.W. (2016) Tree phyllosphere bacterial communities : exploring the magnitude of intra- and inter-individual variation among host species. 4: 1-18.

Langmead, B. and Salzberg, S.L. (2012) Fast gapped-read alignment with Bowtie 2. Nat. Methods 9: 357-359.

Leff, J.W., Del Tredici, P., Friedman, W.E., and Fierer, N. (2015) Spatial structuring of bacterial communities within individual Ginkgo biloba trees. Environ. Microbiol. 17: 2352-2361.

Leppänen, S.M., Rissanen, A.J., and Tiirola, M. (2015) Nitrogen fixation in Sphagnum mosses is affected by moss species and water table level. Plant Soil 389: 185-196.

Li, D., Liu, C.M., Luo, R., Sadakane, K., and Lam, T.W. (2014) MEGAHIT: An ultra-fast single-node solution for large and complex metagenomics assembly via succinct de Bruijn graph. Bioinformatics 31: 1674-1676.

Lindo, Z. and Gonzalez, A. (2010) The Bryosphere: An Integral and Influential Component of the Earth’s Biosphere. 612-627.

Lundberg, D.S., Yourstone, S., Mieczkowski, P., Jones, C.D., and Dangl, J.L. (2013) Practical innovations for high-throughput amplicon sequencing. Nat. Methods 10: 999-1002.

Markowitz, V.M., Chen, I.M.A., Chu, K., Szeto, E., Palaniappan, K., Pillay, M., et al. (2014) IMG/M 4 version of the integrated metagenome comparative analysis system. Nucleic Acids Res. 42: 568573.

Martin, M. (2011) Cutadapt removes adapter sequences from high-throughput sequencing reads. EMBnet.journal 17: 10-12.

McDonald, D., Price, M.N., Goodrich, J., Nawrocki, E.P., DeSantis, T.Z., Probst, A., et al. (2012) An improved Greengenes taxonomy with explicit ranks for ecological and evolutionary analyses of bacteria and archaea. ISME J. 6: 610-618.

Miller, C.S., Baker, B.J., Thomas, B.C., Singer, S.W., and Banfield, J.F. (2011) EMIRGE: reconstruction of full-length ribosomal genes from microbial community short read sequencing data. Genome Biol. 12: R44.

Op den Camp, H.J.M., Islam, T., Stott, M.B., Harhangi, H.R., Hynes, A., Schouten, S., et al. (2009) Environmental, genomic and taxonomic perspectives on methanotrophic Verrucomicrobia. Environ. Microbiol. Rep. 1: 293-306.

Opelt, K., Chobot, V., Hadacek, F., Schönmann, S., Eberl, L., and Berg, G. (2007) Investigations of the structure and function of bacterial communities associated with Sphagnum mosses. Environ. Microbiol. 9: 2795-2809.

Parks, D.H., Imelfort, M., Skennerton, C.T., Hugenholtz, P., and Tyson, G.W. (2015) CheckM: assessing the quality of microbial genomes recovered from isolates, single cells, and metagenomes. Genome Res. 25: 1043-55. 
542

Perovich, D.K. (2007) Light reflection and transmission by a temperate snow cover. J. Glaciol. 53: 201-210.

Price, M.N., Dehal, P.S., and Arkin, A.P. (2010) FastTree 2 - Approximately maximum-likelihood trees for large alignments. PLoS One 5:.

R Core Team (2017) R: A Language and Environment for Statistical Computing.

Redford, A.J., Bowers, R.M., Knight, R., Linhart, Y., and Fierer, N. (2010) The ecology of the phyllosphere: geographic and phylogenetic variability in the distribution of bacteria on tree leaves. Environ. Microbiol. 12: 2885-93.

Rousk, K., Jones, D.L., and Deluca, T.H. (2013) Moss-cyanobacteria associations as biogenic sources of nitrogen in boreal forest ecosystems. 4: 1-10.

Rousk, K., Rousk, J., Jones, D.L., Zackrisson, O., and DeLuca, T.H. (2013) Feather moss nitrogen acquisition across natural fertility gradients in boreal forests. Soil Biol. Biochem. 61: 86-95.

Rousk, K., Sorensen, P.L., Lett, S., and Michelsen, A. (2015) Across-habitat comparison of diazotroph activity in the subarctic. Microb. Ecol. 69: 778-787.

Ruess, R.W., Anderson, M.D., Mcfarland, J.M., Kielland, K., Olson, K., and Taylor, D.L. (2013) Ecosystem-level consequences of symbiont partnerships in an $\mathrm{N}$-fixing shrub from interior Alaskan floodplains. Ecol. Monogr. 83: 177-194.

Ruess, R.W., Mcfarland, J.M., Trummer, L.M., and Rohrs-Richey, J.K. (2009) Disease-mediated declines in N-fixation inputs by Alnus tenuifolia to early-successional floodplains in interior and south-central Alaska. Ecosystems 12: 489-502.

Sharp, C.E., Smirnova, A. V., Graham, J.M., Stott, M.B., Khadka, R., Moore, T.R., et al. (2014) Distribution and diversity of Verrucomicrobia methanotrophs in geothermal and acidic environments. Environ. Microbiol. 16: 1867-1878.

Trexler, R., Solomon, C., Brislawn, C.J., Wright, J.R., Rosenberger, A., Keddache, M., et al. (2014) Assessing impacts of unconventional natural gas extraction on microbial communities in headwater stream ecosystems in Northwestern Pennsylvania. Front. Microbiol. 5: 1-13.

Turetsky, M.R., Bond-Lamberty, B., Euskirchen, E., Talbot, J., Frolking, S., McGuire, a. D., and Tuittila, E.S. (2012) The resilience and functional role of moss in boreal and arctic ecosystems. New Phytol. 196: 49-67.

Vile, M.A., Kelman Wieder, R., Živković, T., Scott, K.D., Vitt, D.H., Hartsock, J.A., et al. (2014) N2fixation by methanotrophs sustains carbon and nitrogen accumulation in pristine peatlands. Biogeochemistry 121: 317-328.

Wang, Q., Garrity, G.M., Tiedje, J.M., and Cole, J.R. (2007) Naïve Bayesian classifier for rapid assignment of rRNA sequences into the new bacterial taxonomy. Appl. Environ. Microbiol. 73: 5261-5267. 
Wu, Y.-W., Tang, Y.-H., Tringe, S.G., Simmons, B. a, and Singer, S.W. (2014) MaxBin: an automated binning method to recover individual genomes from metagenomes using an expectationmaximization algorithm. Microbiome 2: 26.

Yurkov, V. and Hughes, E. (2017) Aerobic Anoxygenic Phototrophs: Four Decades of Mystery. In, Hallenbeck,P.C. (ed), Modern Topics in the Phototrophic Prokaryotes: Environmental and Applied Aspects. Springer International Publishing, Cham, pp. 193-214.Yutin, N., Suzuki, M.T., and Béjà, O. (2005) Novel primers reveal wider diversity among marine aerobic anoxygenic phototrophs. Appl. Environ. Microbiol. 71: 8958-8962.

Yutin, N., Suzuki, M.T., Rosenberg, M., Rotem, D., Madigan, M.T., Süling, J., et al. (2009) BchY-based degenerate primers target all types of anoxygenic photosynthetic bacteria in a single PCR. Appl. Environ. Microbiol. 75: 7556-7559.

Zackrisson, A.O., Deluca, T.H., Nilsson, M., Sellstedt, A., Berglund, L.M., and Zackrisson, O. (2004) Nitrogen fixation increases with successional age in boreal forests. Ecology 85: 3327-3334.

Zackrisson, O., Deluca, T.H., Gentili, F., Sellstedt, A., and Jäderlund, A. (2009) Nitrogen fixation in mixed Hylocomium splendens moss communities. Oecologia 160: 309-319.

\section{Author Contributions}

The project was conceived by N.F., M.C.M, and S.F.M. The manuscript was written by H.H.M. and N.F. with contributions from all co-authors. M.C.M. collected the samples. J.S. and S.M. performed the isotopic analysis. L.R.L. and S.F.M. identified the moss species and prepared the voucher specimens. H.H.M. and N.F. performed the molecular analyses.

\section{Conflict of Interest Statement}

The authors declare no conflict of interest in this study.

\section{Materials and Correspondence}

Requests for materials and correspondence should be addressed to N.F. or H.H.M.

\section{Figure Captions:}

Figure 1: Heat map showing the relative abundances of the most abundant bacterial families across the 7 moss species. Phylum-level classifications for each family are noted on the left. Higher relative abundances are indicated in brighter colors. Families with no official classification are noted as “Unclassified Family.” Host moss species are indicated along the $\mathrm{x}$-axis and ordered according to overall bacterial community similarity.

Figure 2: Non-metric multidimensional scaling (NMDS) plot of bacterial community dissimilarities across samples (measured using the Bray-Curtis distance metric). Samples from the same host species tend to cluster together (A), while those from the same site were spread across ordination space (B). A PERMANOVA test revealed that species identity contributes to $63 \%$ of the variation in community composition $\left(R^{2}=0.63, p<0.001\right)$, while site is not a significant source of variation between these communities $\left(\mathrm{R}^{2}=0.08, \mathrm{p}=0.21\right)$.

Figure 3: A phylogenetic tree of the top 30 bacterial phylotypes (circles) and their $\mathrm{N}_{2}$-fixing potential. Putative $\mathrm{N}_{2}$-fixers (black circles) were identified by their relatedness to known $\mathrm{N}_{2}$-fixers (see text). 
623 The putative $\mathrm{N}_{2}$-fixers observed in our moss samples include representatives from the cyanobacterial 624 and alphaproteobacterial groups. The phylotypes represented here were chosen as they were consistently the most abundant phylotypes across all moss species and together account for 35\% of $16 \mathrm{~S}$ rRNA gene reads in the dataset. Each inner colored ring represents a different species of moss, from the inside ring to the outer ring they are: Sanionia uncinata (blue), Dicranum elongatum (yellow), Aulacomnium turgidum (bright red), Pleurozium schreberi (dark red), Sphagnum capillifolium (green), Aulacomnium palustre (purple), Tomenthypnum nitens (gray). The opacity of the colored rings represents the relative abundance of the different phylotypes (log transformed and scaled between 0 and 1) within each moss species. The black outer ring represents the total relative abundance of each phylotype across all host species (also log transformed and scaled between 0 and 1).

Figure 4: Diagram highlighting the inferred metabolic pathways linked to anoxygenic phototrophy and carbon cycling between the WPS-2 bacterial phylotype found to be abundant in mosses and the moss host cells. In the bacterial cell, light in a complementary spectrum to that absorbed by plants can be used to fuel cellular processes through anoxygenic phototrophy. $\mathrm{CO}_{2}$ fixation via RuBisCo in the bacterial cell may also occur, a novel feature for an aerobic anoxygenic phototroph. In the host cell, photorespiration (the action of RuBisCo on $\mathrm{O}_{2}$ rather than $\mathrm{CO}_{2}$ ) can produce a Calvin Cycle-inhibiting byproduct, 2-phosphoglycolate, that the bacterial cell may have the ability to metabolize for biomass production though conversion to glyoxylate, a process known as the "glyoxylate bypass". This process represents a possible mechanism for a mutalistic or commensal interactions between these WPS-2 bacteria and the moss host. 


\begin{tabular}{|c|c|c|c|c|c|c|c|c|}
\hline \multirow{16}{*}{ Proteobacteria } & Acetobacteraceae- & 0.058 & 0.069 & 0.128 & 0.125 & 0.135 & 0.164 & 0.013 \\
\hline & Sinobacteraceae- & 0.093 & 0.079 & 0.076 & 0.094 & 0.098 & 0.135 & 0.014 \\
\hline & Comamonadaceae- & 0.021 & 0.026 & 0.026 & 0.015 & 0.021 & 0.001 & 0.146 \\
\hline & Caulobacteraceae & 0.015 & 0.015 & 0.028 & 0.031 & 0.028 & 0.026 & 0.015 \\
\hline & Sphingomonadaceae & 0.009 & 0.017 & 0.007 & 0.016 & 0.012 & 0.002 & 0.083 \\
\hline & Unclassified Family $1-$ & 0.031 & 0.018 & 0.019 & 0.012 & 0.004 & 0.017 & 0.003 \\
\hline & Methylocystaceae & 0.015 & 0.019 & 0.009 & 0.023 & 0.018 & 0.009 & 0.003 \\
\hline & Burkholderiaceae- & 0.015 & 0.01 & 0.008 & 0.028 & 0.023 & 0.008 & $<0.005$ \\
\hline & Rhodospirillaceae & 0.015 & 0.012 & 0.025 & 0.01 & 0.01 & 0.014 & 0.004 \\
\hline & Unclassified Family 4 & 0.015 & 0.016 & 0.018 & 0.008 & 0.008 & 0.006 & 0.014 \\
\hline & Unclassified Family 3 & 0.011 & 0.012 & 0.01 & 0.012 & 0.018 & 0.005 & 0.008 \\
\hline & Xanthomonadaceae & 0.027 & 0.015 & 0.008 & 0.01 & 0.003 & 0.005 & 0.007 \\
\hline & Coxiellaceae & 0.012 & 0.006 & 0.016 & 0.013 & 0.003 & 0.022 & 0.001 \\
\hline & Bradyrhizobiaceae- & 0.008 & 0.013 & 0.007 & 0.011 & 0.004 & 0.005 & 0.008 \\
\hline & Unclassified Family 2 & 0.007 & 0.005 & 0.011 & 0.003 & 0.003 & 0.007 & 0.011 \\
\hline & Hyphomicrobiaceae & 0.005 & 0.008 & 0.008 & 0.001 & $<0.005$ & 0.006 & 0.012 \\
\hline \multirow{2}{*}{ Acidobacteria } & Acidobacteriaceae- & 0.063 & 0.056 & 0.052 & 0.129 & 0.158 & 0.122 & 0.006 \\
\hline & Solibacteraceae & 0.018 & 0.014 & 0.021 & 0.02 & 0.016 & 0.03 & 0.002 \\
\hline \multirow{4}{*}{ Verrucomicrobia } & [Chthoniobacteraceae] & 0.034 & 0.029 & 0.037 & 0.051 & 0.051 & 0.015 & 0.049 \\
\hline & Unclassified Family 1 & 0.042 & 0.037 & 0.03 & 0.041 & 0.034 & 0.02 & 0.001 \\
\hline & auto67_4V & 0.032 & 0.022 & 0.02 & 0.003 & $<0.005$ & 0.009 & 0.002 \\
\hline & Opitutaceae- & 0.009 & 0.011 & 0.014 & 0.004 & 0.002 & 0.011 & 0.008 \\
\hline \multirow{3}{*}{ Bacteroidetes } & Chitinophagaceae- & 0.035 & 0.054 & 0.036 & 0.022 & 0.017 & 0.015 & 0.087 \\
\hline & Sphingobacteriaceae & 0.027 & 0.029 & 0.015 & 0.033 & 0.058 & 0.019 & 0.025 \\
\hline & Cytophagacea & 0.005 & 0.011 & 0.011 & 0.001 & $<0.005$ & 0.003 & 0.046 \\
\hline \multirow{2}{*}{ Cyanobacteria } & Nostocaceae- & 0.091 & 0.069 & 0.046 & 0.028 & 0.039 & 0.001 & 0.082 \\
\hline & Unclassified Family 1 & 0.015 & 0.009 & 0.007 & 0.011 & 0.001 & 0.013 & 0.001 \\
\hline WPS-2 & Unclassified Family 1 & 0.064 & 0.065 & 0.06 & 0.077 & 0.075 & 0.062 & 0.001 \\
\hline \multirow{3}{*}{ Planctomycetes } & Unclassified Family 1 & 0.018 & 0.017 & 0.022 & 0.018 & 0.019 & 0.025 & 0.01 \\
\hline & Isosphaeraceae- & 0.013 & 0.016 & 0.012 & 0.022 & 0.03 & 0.023 & 0.002 \\
\hline & Gemmataceae & 0.015 & 0.016 & 0.014 & 0.006 & 0.003 & 0.025 & 0.01 \\
\hline \multirow{2}{*}{ Actinobacteria } & Conexibacteraceae- & 0.004 & 0.005 & 0.001 & 0.012 & 0.011 & 0.03 & $<0.005$ \\
\hline & Microbacteriaceae- & 0.004 & 0.013 & 0.004 & 0.009 & 0.012 & 0.003 & 0.007 \\
\hline
\end{tabular}


bioRxiv preprint doi: https://doi.org/10.1101/219659; this version posted November 16,2017 . The copyright holder for this preprint (which was not certified by peer review) is the author/funder. All rights reserved. No reuse allowed without permission.
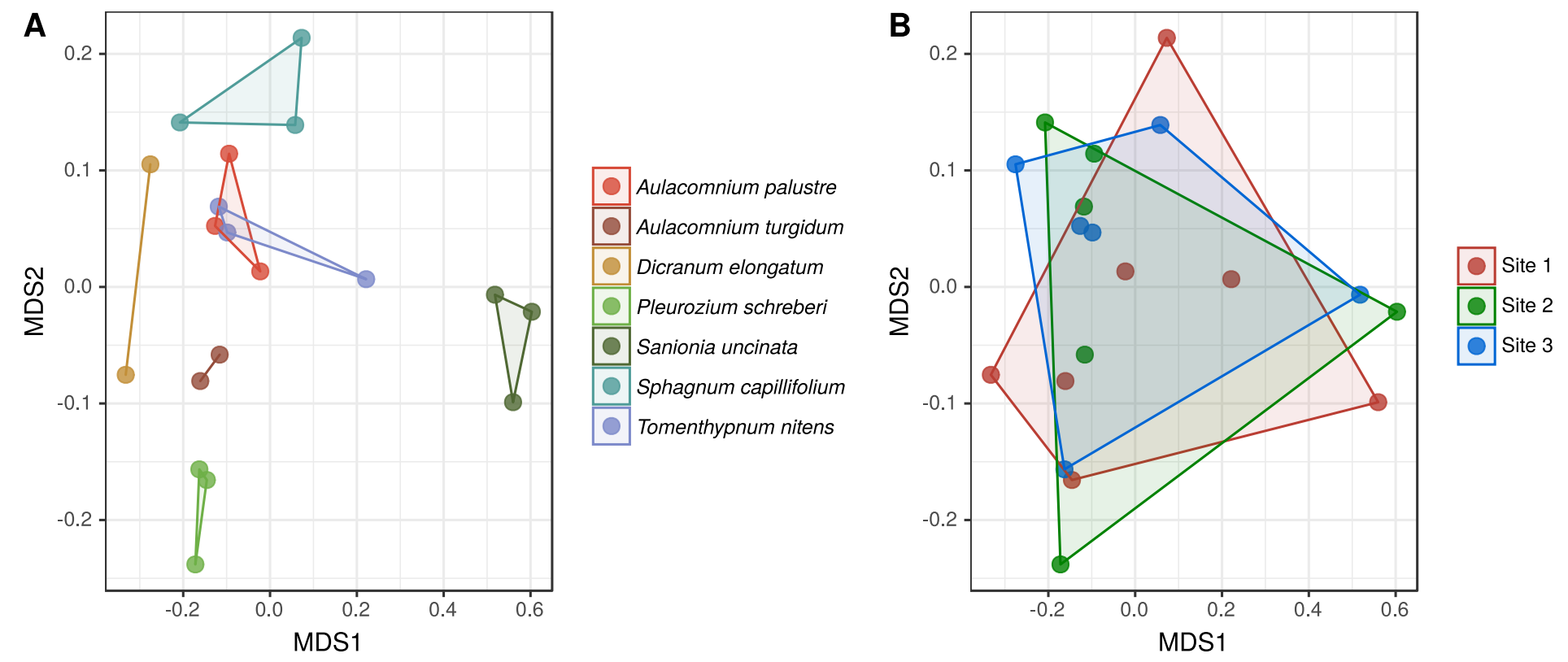


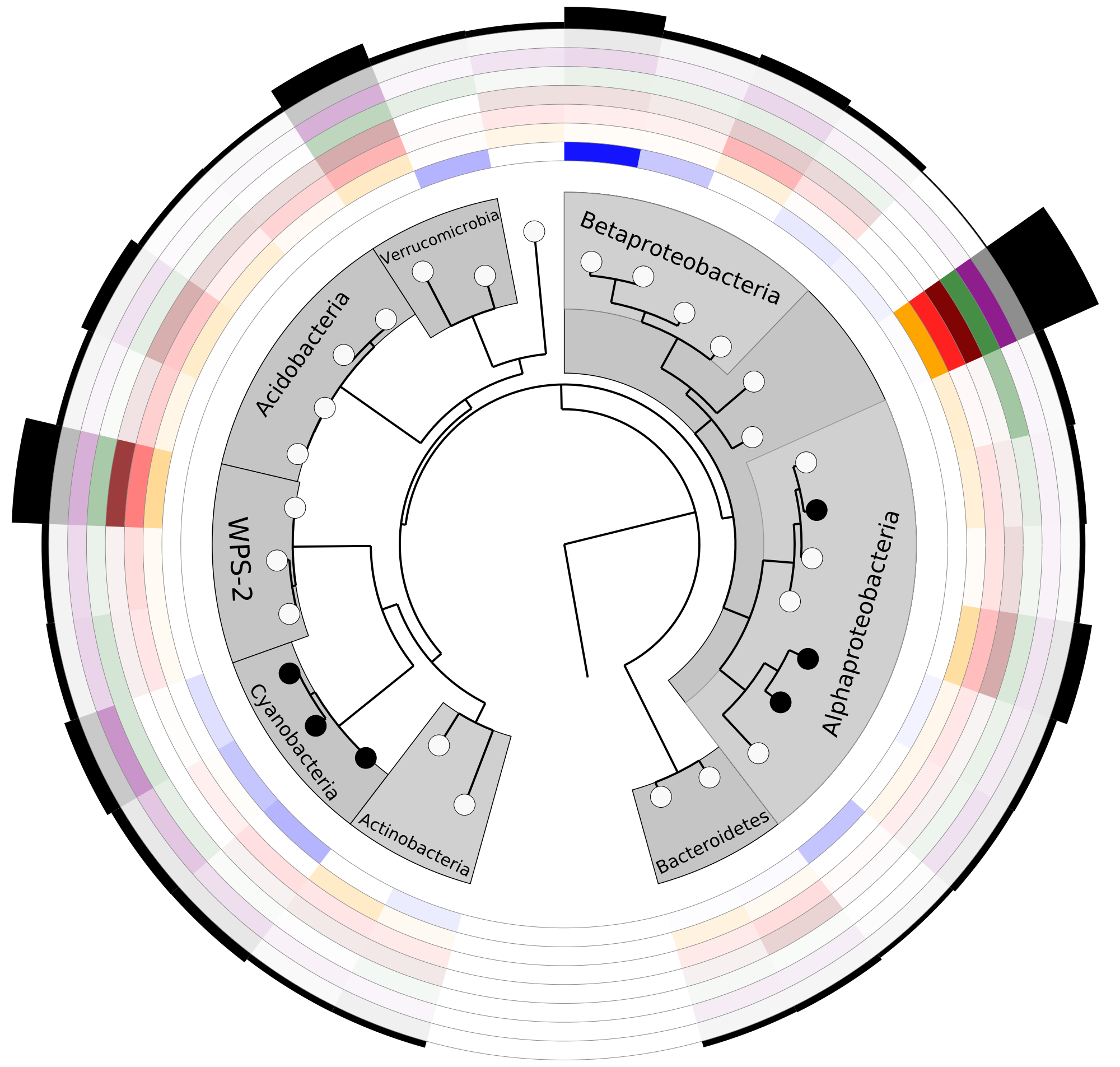

No evidence for $\mathrm{N}_{2}$-fixation

- Putative $\mathrm{N}_{2}$-fixer

Rings

Sanionia uncinata

Dicranum elongatum

Aulacomnium turgidum

Pleurozium schreberi

Sphagnum capillifolium

Aulacomnium palustre

Tomenthypnum nitens

Total Mean Relative Abundance 


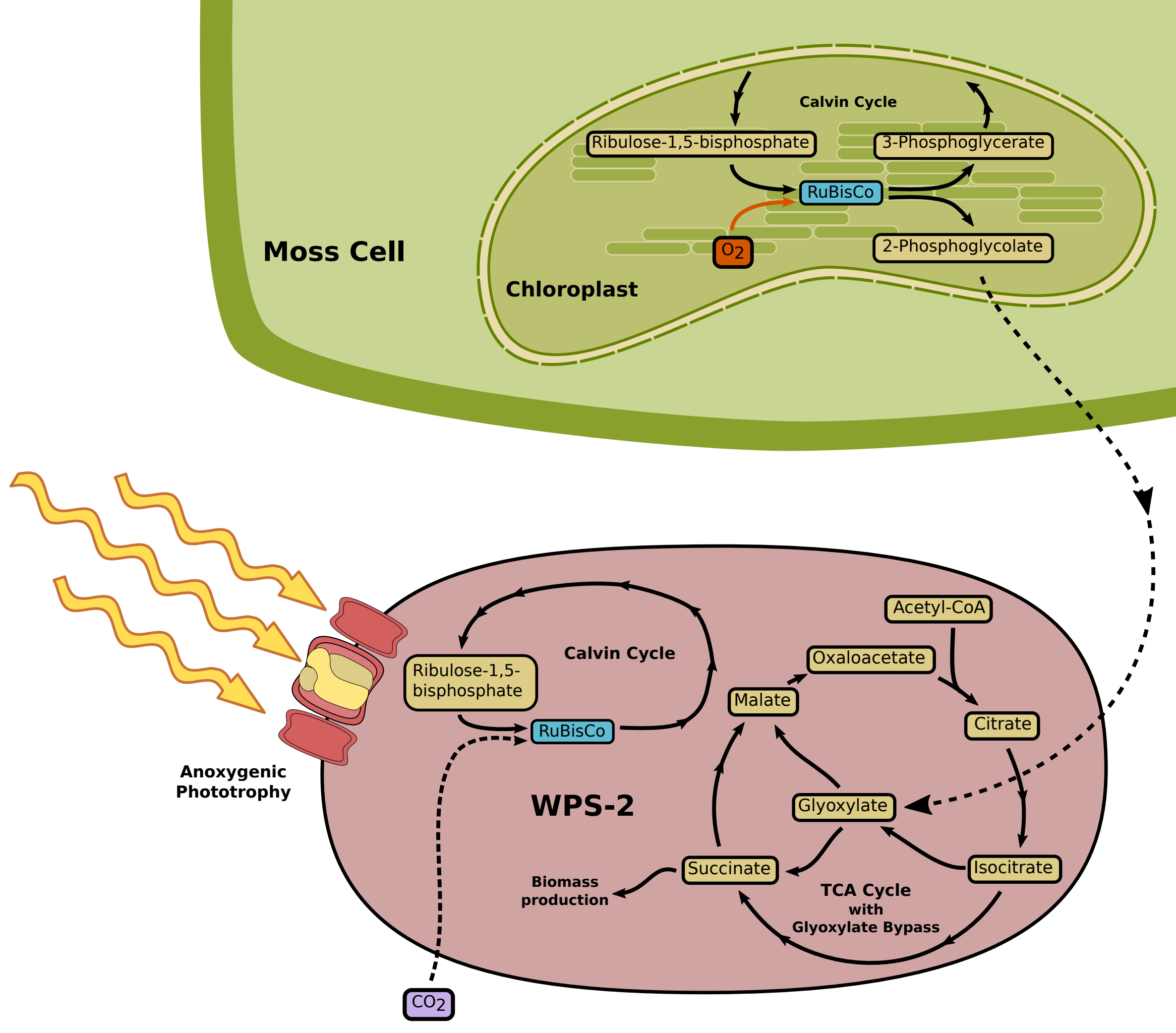

